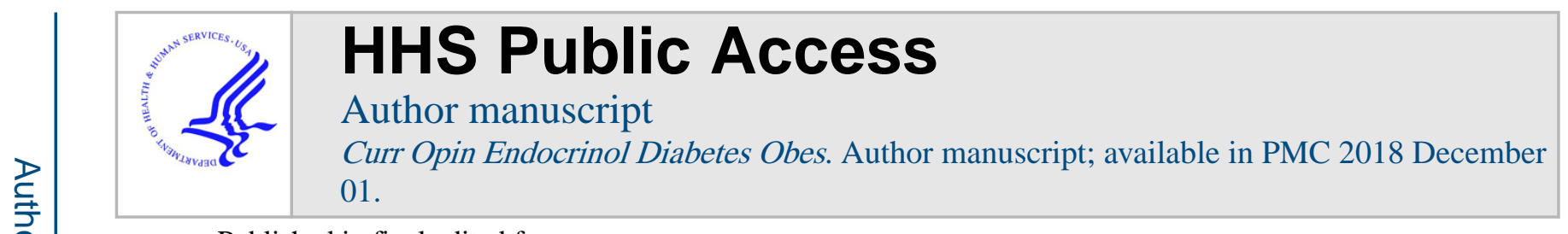

Published in final edited form as:

Curr Opin Endocrinol Diabetes Obes. 2017 December ; 24(6): 411-417. doi:10.1097/MED. 0000000000000368.

\title{
Advances in Treatment of Glucocorticoid-Induced Osteoporosis
}

\author{
Emory Hsu and Mark Nanes \\ Emory University School of Medicine, 101 Woodruff Circle, WMRB 1331, Atlanta GA 30322 USA, \\ 404-727-1398
}

\begin{abstract}
Purpose of Review-To summarize monitoring, prevention, and treatment options of glucocorticoid-induced osteoporosis for patients on chronic glucocorticoid therapy.
\end{abstract}

Recent Findings-Recent meta-analyses highlight the efficacy of bisphosphonate use in improving bone mineral density and in reducing vertebral fractures in the setting of long term glucocorticoid use. A new study has now shown that alendronate also reduces the risk of hip fracture in glucocorticoid use. Emerging data indicate that teriparatide and denosumab also reduce the risk of osteoporotic fracture in glucocorticoid-induced osteoporosis.

Summary-Glucocorticoid use is a leading cause of secondary osteoporosis; however, patients at risk of glucocorticoid-induced osteoporosis are often not evaluated or treated in a timely manner. Patients on a dose equivalent of $2.5 \mathrm{mg}$ prednisone or greater for 3 months or longer duration should have their fracture risk assessed. Those at moderate or high risk should start bisphosphonate therapy, or if contraindicated, a second line agent such as teriparatide or denosumab.

\section{Keywords}

Glucocorticoids; Osteoporosis; Steroids; Bisphosphonates

\section{Introduction}

Osteoporosis is a disease of bone weakness leading to increased fracture risk. As a major cause of morbidity and mortality, it is an important public health concern and serious burden across the world. Secondary osteoporosis is bone loss caused by other medical conditions or treatments including medications. There is a growing list of medications that can result in bone fragility, with glucocorticoids being the most common. Through a combination of their widespread use and their frequent extended duration of use, glucocorticoid induced osteoporosis (GIO) is currently the leading iatrogenic cause of osteoporosis. It is estimated that more than $10 \%$ of patients who receive long-term glucocorticoid treatment are diagnosed with a fracture[1]. Glucocorticoid induced bone loss is most rapid in the first 6 months of steroid use, with a slower decline with chronic use. In addition to using as low of a dose and as short of a duration of glucocorticoids as possible, there are now guidelines for assessing fracture risk and treatments while patients are on glucocorticoids.

Conflicts of interest: None 


\section{Mechanism of Glucocorticoid Action}

A subset of the class of corticosteroids, glucocorticoids are naturally synthesized primarily in the zona fasciculata of the adrenal cortex, or can be administered as pharmacologic agents. Glucocorticoids act by binding to the cytosolic glucocorticoid receptor, which is widely expressed among varying cell types. Upon activation of the glucocorticoid receptor, the complex translocates to the nucleus, where it binds glucocorticoid response elements of an array of genes. This in turn modulates transcription of target genes, resulting in expression of enzymes crucial for normal cellular functions such as gluconeogenesis and anti-inflammation. As such, hydrocortisone is one of the most important hormones in the human body. Non-canonical pathways play less important roles, but these nongenomic effects have also been shown to contribute to various mechanisms of downregulating inflammatory markers[2].

In terms of the direct impact on bone, endogenous glucocorticoids at physiologic concentrations may have a role in promoting osteogenesis[3], while excess glucocorticoids increase osteoclastogenesis and suppress osteblastogenesis in cell culture, murine, and human models $[4,5]$. Local metabolism of glucocorticoids in bone cells is controlled by a pair of complementary enzymes, 11ß-hydroxysteroid dehydrogenase types 1 and 2 (Hsd11b1 \& Hsd11b2), which respectively activate or deactivate glucocorticoid action by metabolizing the interconversion of biologically active or inert forms[6, 7]. Additional pathways of glucocorticoid action are thought to be multiple, including inducing proapoptotic molecules in osteoblasts and osteocytes, and through antagonizing the osteoblastogenic Wnt pathway $[4,8,9]$. There continues to be active work into uncovering pathways of glucocorticoid mechanism at the cellular level[10], including recent claims of enhancing osteoblast activity through heat shock protein 90[11].

In addition, there are indirect effects of glucocorticoids on skeletal health. They suppress IGF-1, a hormone crucial for general growth [12] and bone and collagen formation [13]. They inhibit calcium absorption from the intestine. Effects of glucocorticoids on suppressing estrogen and testosterone production may also augment deleterious effects on bone. Finally, many of the underlying disease states for which chronic glucocorticoids are prescribed are themselves linked to lower bone mineral density (BMD). Patients with Crohn's or cystic fibrosis are at risk of bone fragility due to malabsorption, and many conditions of chronic inflammation, such as rheumatoid arthritis or inflammatory bowel diseases, are associated with systemic increases in cytokines such as IL-6, RANKL, and IL-17, which drive the skeletal balance toward osteoclastogenesis [14].

\section{Pharmacologic Preparations of Glucocorticoids}

Glucocorticoids are transported in the bloodstream bound to corticosteroid-binding globulin and albumin, in equilibrium with the biologically active free form that binds to the glucocorticoid receptor. Individual sensitivity to glucocorticoid signaling is also affected by genetic variability of the glucocorticoid receptor, with alternative splicing and polymorphisms identified [15, 16]. Furthermore, pharmaceutical preparations have differences in absorption, transport, and target affinity, and thus a varied range of potencies 
and duration of effects. Glucocorticoid potency ranges from lower potency such as with cortisone and prednisone, to higher potency, such as with dexamethasone and betamethasone. In general, when oral cortisol (hydrocortisone) is used as a baseline, prednisone and prednisolone have about four times the potency, while methylprednisolone and triamcinolone are about five times the potency, and dexamethasone and betamethasone are about twenty-five times the potency. Reference charts of glucocorticoid potencies can be easily obtained [17].

Most common forms of administered glucocorticoids are oral preparations. Intravenous, inhaled, injected, and transdermal preparations are also frequently encountered. However, side effects, including to bone, are not limited to oral or intravenous administrations. Injected glucocorticoids, particularly when repeated, and topical therapy may both lead to systemic effects.

\section{Clinical Course of Glucocorticoid-Induced Osteoporosis}

The primary difference between GIO and post-menopausal osteoporosis is the suppression of osteoblastic activity, leading to decreased bone formation. However, an early phase consists of rapid loss of bone mineral density due mostly to excessive bone resorption, while impaired bone formation usually manifests more progressively with long-term therapy. Trabecular bone loss predominates, with most marked changes in the lumbar spine, but also in the femoral neck and other sites. The effect on bone is dose dependent, with relative risk significantly increased at daily doses higher than $2.5 \mathrm{mg}$ prednisolone equivalent [18]. It is also dependent on duration, with fracture risk returning to baseline months after cessation of therapy. However, changes to bone can occur sooner than many physicians realize. One study found that a 40mg or higher prednisone equivalent a day can result in substantial BMD loss at the lumbar spine in just two months. [19] Meta-analyses indicate that exposure to steroid use is associated with a relative risk of fracture of about 1.6 to 1.98 , and is independent of gender $[20,21]$.

Fractures most often occur at sites enriched with trabecular (cancellous) bone, such as the lumbar spine and femoral neck. Vertebral fractures may be asymptomatic and detected only by radiographic imaging. While much of the knowledge of GIO is extrapolated from experience with postmenopausal osteoporosis, it is important to note that fractures tend to occur at higher bone mineral density in glucocorticoid induced osteoporosis [22].

\section{Risk Stratification, Screening and Assessment}

Management of patients who remain on glucocorticoids is based on risk modification and prevention of osteoporosis. The rate of screening for bone disease has traditionally varied by the medical specialty of the provider prescribing the chronic glucocorticoid [23], but awareness has increased in recent years [24]. There are several groups with recommendations for identifying high risk of fracture in patients on glucocorticoids. Most determine an individual intervention threshold based on calculating a probability of fracture through using the Fracture Risk Assessment Tool score (FRAX), although there are other models [25]. The FRAX model has a built-in adjustment for glucocorticoid use. However, 
the BMD used in the FRAX tool is the femoral neck, while GIO more heavily affects sites with more trabecular bone such as the lumbar spine, so FRAX will underestimate the probability of fracture. Corroborating this, a study in Taiwan noted that glucocorticoid users have a higher probability of major osteoporotic fracture regardless of bone mineral density [26]. Furthermore, FRAX cannot be used in people less than 40 years of age, so clinical judgment is required. Thus, adults younger than age 40 do not need BMD measurement unless a history of previous osteoporotic fracture or other risk factors are present, while all patients on chronic glucocorticoids over the age of 40 should have BMD and FRAX calculation within 6 months of initiation of glucocorticoids. There are no clear data on frequency of BMD screening, but in general, BMD can be reassessed based on the rate of bone loss of each patient. Patients recently starting glucocorticoids or those with multiple other risk factors or a history of fracture may need BMD measurement every 6 months, while those with more stability may have BMD measured as intermittently as once every three years.

Fracture risk as defined by the 2017 American College of Rheumatology Guidelines [27] is calculated for those on prednisone equivalent of $2.5-7.5 \mathrm{mg} /$ day, while those receiving higher doses should increase their major fracture risk by $15 \%$ and by $20 \%$ for the hip. For adults over age 40, the high risk group is defined by those who have had prior osteoporotic fracture, or a total FRAX 10-year risk of $>20 \%$ major fracture or $>3 \%$ hip fracture, or for postmenopausal women and men over age 50, a hip or spine BMD T score $<-2.5$. Moderate risk comprises those with 10-year major fracture risk of 10-19\% or hip fracture risk of 1$3 \%$, and low risk are those with $<10 \%$ total and $<1 \%$ hip fracture risks. For adults younger than age 40, high risk is any previous history of osteoporotic fracture, while moderate risk is a hip or spine BMD Z score of $<-3$ or rapid bone loss of over $10 \%$ in the past year, in addition to high dose chronic steroid use of $>7.5 \mathrm{mg}$ prednisone equivalent a day for greater than 6 months. These younger patients are otherwise considered low risk. (Figure 1)

\section{Treatment of Patients on Chronic Glucocorticoids}

All patients on glucocorticoids should have an adequate intake of vitamin D and calcium, with supplementation given if needed. Serum vitamin D levels should be maintained at a minimum of $20 \mathrm{ng} / \mathrm{mL}$, if not higher. Lifestyle modifications are similar to those for postmenopausal osteoporosis, and include weight-bearing exercise and elimination of other risk factors such as smoking or alcohol abuse. The need for additional treatment is based on the risk stratification discussed above. Patients at moderate or high risk of osteoporosis are recommended to start pharmacologic agents. There is a plethora of evidence to support pharmacologic treatment in both men and women at high risk of glucocorticoid induced osteoporosis. The first line agent has been, and remains, bisphosphonate therapy. If bisphosphonates are contraindicated, teriparatide is a suggested second line agent, followed by denosumab.

Bisphosphonates, such as once weekly oral alendronate or once monthly oral ibandronate, have been the most studied agent for prevention and treatment of GIO. Most studies have focused on vertebral fractures as a primary endpoint, though bone mineral density is frequently a proxy. A 2016 Cochrane review of 27 randomized control trials for 
bisphosphonates in chronic glucocorticoid users found a high certainty of evidence that bisphosphonates are beneficial in reducing the risk of vertebral fractures at 24 months of use, and moderate-certainty evidence of preventing bone loss at the lumbar spine and femoral neck. [28] Several smaller studies published since then have also corroborated the efficacy of bisphosphonates in reducing bone mineral loss [29, 30].

In addition to improving BMD or vertebral fracture risk, treatment can also reduce the risk of hip fractures. In a Swedish retrospective cohort study of patients aged 65 or older who were on 3 months or more of at least $5 \mathrm{mg}$ /day oral prednisolone, alendronate treatment resulted in a lower risk of hip fracture compared to no bisphosphonate treatment [31].

Teriparatide (a recombinant PTH) has also been shown to reduce fracture risk-in fact, proving superior to alendronate in a comparison trial [32]. Another 2016 meta-analysis also found that teriparatide (as well as certain bisphosphonates) were associated with decreased vertebral fracture risk [33]. In theory, as glucocorticoids do have a suppressive effect on osteoblasts, teriparatide would be an appropriate countermeasure as an anabolic agent. However, due to higher cost and inconvenience of administration, teriparatide remains in practice largely a second line agent behind bisphosphonates in treating patients who are on glucocorticoids.

Denosumab, a RANKL inhibitor, has also been considered for prevention of glucocorticoid induced osteoporosis. Denosumab is given as a subcutaneous injection every six months, which may result in better adherence than oral bisphosphonate therapy [34]. However, data is more limited than that for use of bisphosphonates for GIO. Despite its proven efficacy in postmenopausal women [35], there remain few studies specifically looking at GIO. One study of concurrent glucocorticoid and denosumab use in rheumatoid arthritis patients found increased lumbar spine and hip bone mineral density[36]. A few small prospective studies of patients on glucocorticoids from Japan have found that lumbar spine, but not hip, BMD increased with denosumab treatment [37, 38]. In addition, a small study has shown preliminary data that switching from bisphosphonate to denosumab results in increased BMD while on long term steroids, though the sample size was small and clinical fracture data was lacking [39]. Finally, care must be taken in patients with organ transplant (a common indication of chronic glucocorticoid use), as there is insufficient data on safety of denosumab in patients with multiple immunosuppressive agents.

\section{Special Consideration: Children on Steroids}

Children represent a different approach as bone development is still critical. In children, weight gain, growth retardation, and Cushingoid features are the most frequent adverse reaction to chronic oral glucocorticoid use, but a recent meta-analysis that included a total of 6817 children noted a $21 \%$ incidence of decreased bone mineral density [40]. Growth retardation, whether directly or through suppression of the hypothalamic-pituitary axis or of sex steroid hormone production, puts children at considerable risk of osteoporosis in adulthood, as peak bone mass is achieved in late adolescence and early adulthood. While there are few prospective randomized controlled trials, there is general consensus for ensuring adequate calcium and vitamin $\mathrm{D}$ intake and for avoidance of further pharmacologic 
means such as bisphosphonates. Bisphosphonate use should only be considered, and even then very carefully, in children who have an osteoporotic fracture who are still continuing long-term glucocorticoid use.

For inhaled steroid use in children, commonly prescribed for asthma, most studies have not found effects of inhaled steroid use on bone markers[41-43], although a few have identified decreased BMD with high dose inhaled corticosteroids[44]. It is unclear if there is confounding as better control of asthma leads to greater physical activity, which is beneficial to bone development. It is now well-accepted that no bone-specific monitoring or pharmacologic treatment is needed in intermittent or routine inhaled corticosteroid use for asthma, though "periodic" evaluations of bone density may be advised in long-term, highdose therapies [45].

\section{Future Directions}

In addition to solidifying evidence on teriparatide and denosumab use, there remain several avenues of work for alternate means of prevention and treatment of GIO. In a mouse model, activating Wnt/beta-catenin signaling through a sclerostin knockout protected against the skeletal actions of excess glucocorticoids, hinting that sclerostin inhibitors may improve bone integrity in glucocorticoid use [46]. An anti-sclerostin antibody, romosozumab, recently completed phase III trials for use in postmenopausal osteoporosis, but has not come to market because of adverse effect safety concerns[47].

Additionally, the interplay between gut microbiota and bone remodeling has come to light. Recent studies have shown that microbiota affect bone physiology [48], and that supplementation with probiotics can prevent estrogen-deficiency induced osteoporosis in mice $[49,50]$. A putative mechanism is through modulating intestinal permeability and thus $\mathrm{T}$ cell and inflammatory cytokine profile. If ongoing human trials can replicate an effect, the use of probiotics could be a low-cost method to prevent bone loss with few adverse effects, although whether or not its mechanism could be applied to glucocorticoid-induced osteoporosis is yet to be determined.

\section{Conclusions}

Glucocorticoid use of over 3 months duration leads to decreased bone mineral density, primarily through osteoblast suppression, as well as through increasing osteoclast life span. Awareness of osteoporosis risk mandates the stratification of patients into low, moderate, and high risk categories. All patients should maintain adequate intake of calcium and vitamin $\mathrm{D}$, while those in moderate and high risk categories should initiate bisphosphonate therapy, or if bisphosphonates are contraindicated, use of alternative agents such as teriparatide or denosumab.

\section{Acknowledgments}

Figure illustration by Pauline Lu.

Financial support and sponsorship: None 


\title{
Abbreviations
}

\author{
BMD Bone mineral density \\ GIO glucocorticoid-induced osteoporosis \\ FRAX Fracture Risk Assessment Tool score
}

\section{References}

1. Rizzoli R, Biver E. Glucocorticoid-induced osteoporosis: who to treat with what agent? Nat Rev Rheumatol. 2015; 11:98-109. [PubMed: 25385412]

2. Hofbauer LC, Rauner M. Minireview: live and let die: molecular effects of glucocorticoids on bone cells. Molecular endocrinology (Baltimore, Md). 2009; 23:1525-1531.

3. Kalak R, Zhou H, Street J, et al. Endogenous glucocorticoid signalling in osteoblasts is necessary to maintain normal bone structure in mice. Bone. 2009; 45:61-67. [PubMed: 19358901]

4. Komori T. Glucocorticoid Signaling and Bone Biology. Hormone and metabolic research = Hormon- und Stoffwechselforschung $=$ Hormones et metabolisme. 2016; 48:755-763. [PubMed: 27871116]

5. Canalis E, Mazziotti G, Giustina A, Bilezikian JP. Glucocorticoid-induced osteoporosis: pathophysiology and therapy. Osteoporosis international: a journal established as result of cooperation between the European Foundation for Osteoporosis and the National Osteoporosis Foundation of the USA. 2007; 18:1319-1328.

6. Sher LB, Harrison JR, Adams DJ, Kream BE. Impaired cortical bone acquisition and osteoblast differentiation in mice with osteoblast-targeted disruption of glucocorticoid signaling. Calcified tissue international. 2006; 79:118-125. [PubMed: 16927049]

7. Sher LB, Woitge HW, Adams DJ, et al. Transgenic expression of 11 beta-hydroxysteroid dehydrogenase type 2 in osteoblasts reveals an anabolic role for endogenous glucocorticoids in bone. Endocrinology. 2004; 145:922-929. [PubMed: 14617568]

8. Sato AY, Richardson D, Cregor M, et al. Glucocorticoids Induce Bone and Muscle Atrophy by Tissue-Specific Mechanisms Upstream of E3 Ubiquitin Ligases. Endocrinology. 2017; 158:664677. [PubMed: 28359087]

9. Geurtzen K, Vernet A, Freidin A, et al. Immune suppressive and bone inhibitory effects of prednisolone in growing and regenerating zebrafish tissues. Journal of bone and mineral research : the official journal of the American Society for Bone and Mineral Research. 2017

10. Yang N, Baban B, Isales CM, Shi XM. Role of glucocorticoid-induced leucine zipper (GILZ) in inflammatory bone loss. 2017; 12:e0181133.

11. Chen H, Xing J, Hu X, et al. Inhibition of heat shock protein 90 rescues glucocorticoid-induced bone loss through enhancing bone formation. The Journal of steroid biochemistry and molecular biology. 2017; 171:236-246. [PubMed: 28408351]

12. Mazziotti G, Formenti AM, Adler RA, et al. Glucocorticoid-induced osteoporosis: pathophysiological role of GH/IGF-I and PTH/VITAMIN D axes, treatment options and guidelines. Endocrine. 2016; 54:603-611. [PubMed: 27766553]

13. Canalis E, Centrella M, Burch W, McCarthy TL. Insulin-like growth factor I mediates selective anabolic effects of parathyroid hormone in bone cultures. The Journal of clinical investigation. 1989; 83:60-65. [PubMed: 2910920]

14. Pacifici R. Role of T cells in the modulation of PTH action: physiological and clinical significance. Endocrine. 2013; 44:576-582. [PubMed: 23729167]

15. Song QQ, Xie WY, Tang YJ, et al. Genetic variation in the glucocorticoid pathway involved in interindividual differences in the glucocorticoid treatment. Pharmacogenomics. 2017; 18:293-316. [PubMed: 28112586]

16. Whirledge SD, Jewell CM, Barber LM, et al. Generating diversity in human glucocorticoid signaling through a racially diverse polymorphism in the beta isoform of the glucocorticoid receptor. Lab Invest. 2017 
17. Caplan A, Fett N, Rosenbach M, et al. Prevention and management of glucocorticoid-induced side effects: A comprehensive review: A review of glucocorticoid pharmacology and bone health. Journal of the American Academy of Dermatology. 2017; 76:1-9. [PubMed: 27986132]

18. Van Staa TP, Leufkens HG, Abenhaim L, et al. Use of oral corticosteroids and risk of fractures. Journal of bone and mineral research : the official journal of the American Society for Bone and Mineral Research. 2000; 15:993-1000.

19. Natsui K, Tanaka K, Suda M, et al. High-dose glucocorticoid treatment induces rapid loss of trabecular bone mineral density and lean body mass. Osteoporosis international : a journal established as result of cooperation between the European Foundation for Osteoporosis and the National Osteoporosis Foundation of the USA. 2006; 17:105-108.

20. Kanis JA, Johansson H, Oden A, et al. A meta-analysis of prior corticosteroid use and fracture risk. Journal of bone and mineral research : the official journal of the American Society for Bone and Mineral Research. 2004; 19:893-899.

21. Steinbuch M, Youket TE, Cohen S. Oral glucocorticoid use is associated with an increased risk of fracture. Osteoporosis international : a journal established as result of cooperation between the European Foundation for Osteoporosis and the National Osteoporosis Foundation of the USA 2004;. 15:323-328.

22. Van Staa TP, Laan RF, Barton IP, et al. Bone density threshold and other predictors of vertebral fracture in patients receiving oral glucocorticoid therapy. Arthritis and rheumatism. 2003; 48:3224-3229. [PubMed: 14613287]

23. Curtis JR, Westfall AO, Allison JJ, et al. Longitudinal patterns in the prevention of osteoporosis in glucocorticoid-treated patients. Arthritis and rheumatism. 2005; 52:2485-2494. [PubMed: 16052570]

24. Weinstein RS. Clinical practice. Glucocorticoid-induced bone disease The New England journal of medicine. 2011; 365:62-70. [PubMed: 21732837]

25. Francesco L, Elisa B, Raffaella M, et al. Assessing Risk of Osteoporotic Fractures in Primary Care: Development and Validation of the FRA-HS Algorithm. Calcified tissue international. 2017; 100:537-549. [PubMed: 28160026]

26. Yu SF, Chen JF, Chen YC, et al. Beyond bone mineral density, FRAX-based tailor-made intervention thresholds for therapeutic decision in subjects on glucocorticoid: A nationwide osteoporosis survey. Medicine. 2017; 96:e5959. [PubMed: 28151883]

27. Buckley L, Guyatt G, Fink HA, et al. 2017 American College of Rheumatology Guideline for the Prevention and Treatment of Glucocorticoid-Induced Osteoporosis. Arthritis \& rheumatology (Hoboken, N.J.). 2017; 69:1521-1537. Revised society guidelines that can help determine how to monitor and treat patients on steroids. This is one of the most respected consensus groups on this topic.

28. Allen CS, Yeung JH, Vandermeer B, Homik J. Bisphosphonates for steroid-induced osteoporosis. The Cochrane database of systematic reviews. 2016; 10:Cd001347. This is a Cochrane review, one of the largest retrospective meta-analysis done to look at bisphosphonate use specifically in glucocorticoid-induced osteoporosis. [PubMed: 27706804]

29. Shin K, Park SH, Park W, et al. Monthly Oral Ibandronate Reduces Bone Loss in Korean Women With Rheumatoid Arthritis and Osteopenia Receiving Long-term Glucocorticoids: A 48-week Double-blinded Randomized Placebo-controlled Investigator-initiated Trial. Clinical therapeutics. 2017; 39:268-278.e262. [PubMed: 28161119]

30. Tanaka Y, Mori H, Aoki T, et al. Analysis of bone metabolism during early stage and clinical benefits of early intervention with alendronate in patients with systemic rheumatic diseases treated with high-dose glucocorticoid: Early DIagnosis and Treatment of OsteopoRosis in Japan (EDITOR-J) study. Journal of bone and mineral metabolism. 2016; 34:646-654. [PubMed: 26308708]

31. Axelsson KF, Nilsson AG, Wedel H, et al. Association between alendronate use and hip fracture risk in older patients using oral prednisolone. Jama. 2017; 318:146-155. This is the first major article to show that bisphosphonates reduce the risk of hip fracture in glucocorticoid use. Most of the previous literature looked at vertebral fractures or at bone mineral density. [PubMed: 28697254] 
32. Saag KG, Shane E, Boonen S, et al. Teriparatide or alendronate in glucocorticoid-induced osteoporosis. The New England journal of medicine. 2007; 357:2028-2039. [PubMed: 18003959]

33. Amiche MA, Albaum JM, Tadrous M, et al. Efficacy of osteoporosis pharmacotherapies in preventing fracture among oral glucocorticoid users: a network meta-analysis. Osteoporosis international : a journal established as result of cooperation between the European Foundation for Osteoporosis and the National Osteoporosis Foundation of the USA. 2016; 27:1989-1998. Another meta-analysis looking at bisphosphonates, teriparatide, and denosumab in preventing glucocorticoid induced osteoporosis.

34. Reyes C, Tebe C, Martinez-Laguna D, et al. One and two-year persistence with different antiosteoporosis medications: a retrospective cohort study. Osteoporosis international : a journal established as result of cooperation between the European Foundation for Osteoporosis and the National Osteoporosis Foundation of the USA. 2017

35. McClung MR, Lewiecki EM, Cohen SB, et al. Denosumab in postmenopausal women with low bone mineral density. The New England journal of medicine. 2006; 354:821-831. [PubMed: 16495394]

36. Dore RK, Cohen SB, Lane NE, et al. Effects of denosumab on bone mineral density and bone turnover in patients with rheumatoid arthritis receiving concurrent glucocorticoids or bisphosphonates. Annals of the rheumatic diseases. 2010; 69:872-875. [PubMed: 19734132]

37. Ishiguro S, Ito K, Nakagawa S, et al. The clinical benefits of denosumab for prophylaxis of steroidinduced osteoporosis in patients with pulmonary disease. Archives of osteoporosis. 2017; 12:44. A small study of denosumab specifically regarding glucocorticoid induced osteoporosis that adds to the nascent but growing data showing denosumab is effective in GIO. [PubMed: 28425086]

38. Sawamura M, Komatsuda A, Togashi M, et al. Effects of Denosumab on Bone Metabolic Markers and Bone Mineral Density in Patients Treated with Glucocorticoids. Internal medicine (Tokyo, Japan). 2017; 56:631-636.

39. Mok CC, Ho LY, Ma KM. Switching of oral bisphosphonates to denosumab in chronic glucocorticoid users: a 12-month randomized controlled trial. Bone. 2015; 75:222-228. [PubMed: 25761434]

40. Aljebab F, Choonara I, Conroy S. Systematic Review of the Toxicity of Long-Course Oral Corticosteroids in Children. PloS one. 2017; 12:e170259. This review looks at children, and different forms of steroids and their adverse effects, not limited to bone. Treatment of children is complicated by the need for their bones to continue growing. [PubMed: 28125632]

41. Roux C, Kolta S, Desfougeres JL, et al. Long-term safety of fluticasone propionate and nedocromil sodium on bone in children with asthma. Pediatrics. 2003; 111:e706-713. [PubMed: 12777589]

42. Szefler S, Weiss S, Tonascia J, et al. Long-term effects of budesonide or nedocromil in children with asthma. The New England journal of medicine. 2000; 343:1054-1063. [PubMed: 11027739]

43. Zieck SE, George J, Blakeley BA, et al. Asthma, bones and corticosteroids: Are inhaled corticosteroids associated with fractures in children with asthma? Journal of paediatrics and child health. 2017; 53:771-777. [PubMed: 28568681]

44. Skoner DP. Inhaled corticosteroids: Effects on growth and bone health. Annals of allergy, asthma \& immunology : official publication of the American College of Allergy, Asthma, \& Immunology. 2016; 117:595-600.

45. Wolfgram PM, Allen DB. Effects of Inhaled Corticosteroids on Growth, Bone Metabolism, and Adrenal Function. Advances in pediatrics. 2017; 64:331-345. [PubMed: 28688596]

46. Sato AY, Cregor M, Delgado-Calle J, et al. Protection From Glucocorticoid-Induced Osteoporosis by Anti-Catabolic Signaling in the Absence of Sost/Sclerostin. Journal of bone and mineral research : the official journal of the American Society for Bone and Mineral Research. 2016; 31:1791-1802.

47. Cosman F, Crittenden DB, Adachi JD, et al. Romosozumab Treatment in Postmenopausal Women with Osteoporosis. New England Journal of Medicine. 2016; 375:1532-1543. [PubMed: 27641143]

48. Sjogren K, Engdahl C, Henning P, et al. The gut microbiota regulates bone mass in mice. Journal of bone and mineral research : the official journal of the American Society for Bone and Mineral Research. 2012; 27:1357-1367. 
49. Britton RA, Irwin R, Quach D, et al. Probiotic L. reuteri treatment prevents bone loss in a menopausal ovariectomized mouse model. Journal of cellular physiology. 2014; 229:1822-1830. [PubMed: 24677054]

50. Li JY, Chassaing B, Tyagi AM, et al. Sex steroid deficiency-associated bone loss is microbiota dependent and prevented by probiotics. The Journal of clinical investigation. 2016; 126:20492063. This study definitively demonstrates the key role that microbiota play in maintaining bone homeostasis including in estrogen deficiency, and shows that probiotics can prevent osteoporosis in mice. Microbiota manipulation through prebiotics, probiotics, antibiotics, or other means, has become a hot topic and may result in new therapeutics for osteoporosis. [PubMed: 27111232] 


\section{Key Points}

- $\quad$ Extra-physiologic doses of glucocorticoids suppress osteoblastogenesis and alter osteoclast activity

- Use of glucocorticoids at a dose equivalent of $2.5 \mathrm{mg}$ of prednisone or greater for 3 months or longer leads to an increased fracture risk not completely reflected in bone mineral density measurement or $\mathrm{T}$ score, as glucocorticoids cause a disproportionate impairment in skeletal microarchitecture and quality

- A prior osteoporotic fracture, a T score of less than -2.5 in the hip or spine for men over age 50 or postmenopausal women, or a 10 -year estimated risk of greater than $10 \%$ major osteoporotic fracture or $1 \%$ hip fracture based on FRAX, are qualifications for moderate to high risk.

- For adults less than 40 years old, a $\mathrm{Z}$ score of less than -3 at the hip or spine, or rapid bone loss of more than $10 \%$ over one year, in addition to higher dose glucocorticoid use of greater than $7.5 \mathrm{mg}$ equivalent a day, are qualifications for moderate risk.

- $\quad$ Evidence supports use of bisphosphonates, teriparatide, or denosumab in adults with moderate to high risk on chronic glucocorticoids 


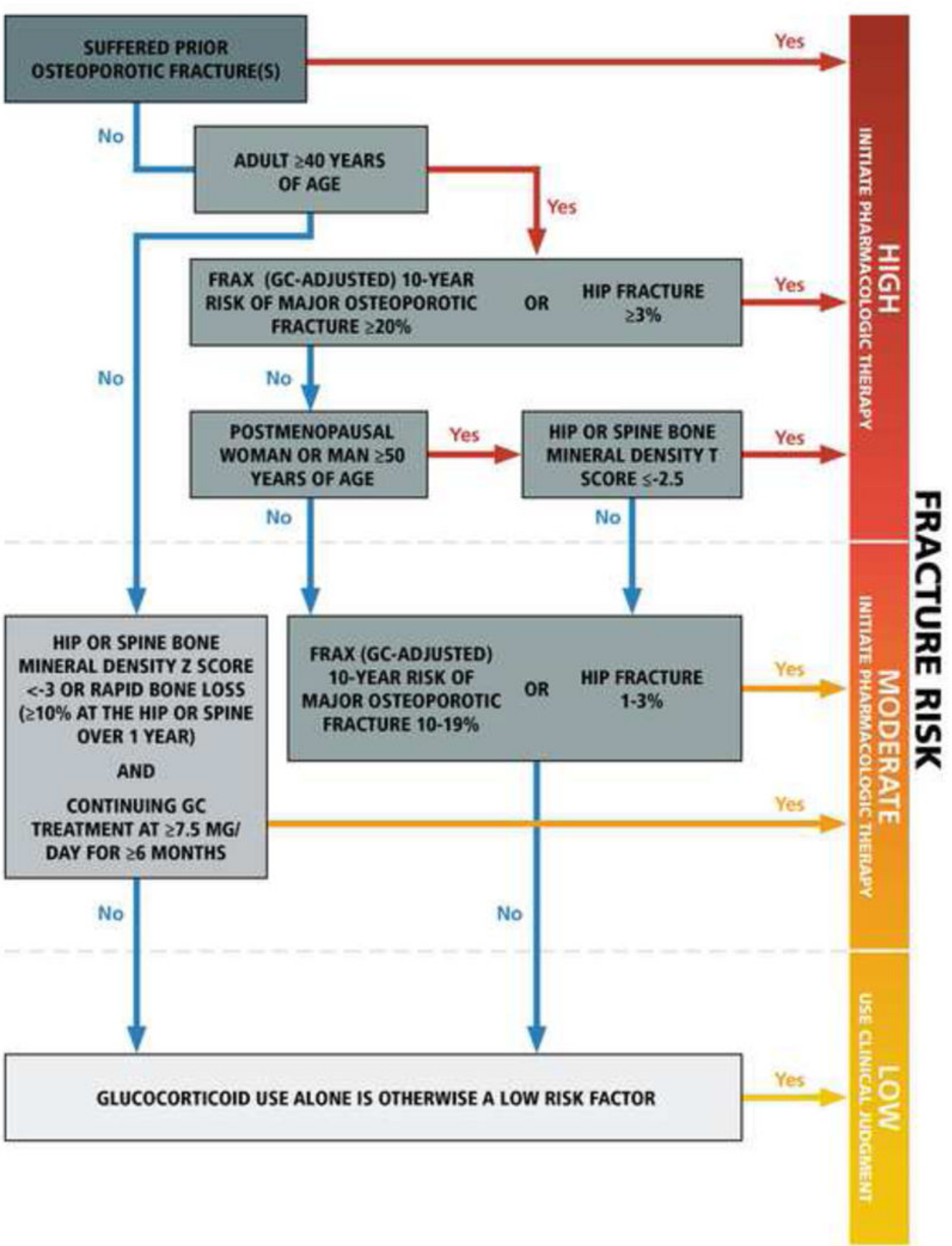

Figure 1. Risk Assessment

Flow-chart for stratifying fracture risk in adult patients on chronic glucocorticoids (prednisone at $>2.5 \mathrm{mg} /$ day for $\geq 3$ months) without impaired renal function (glomerular filtration rate of $<30 \mathrm{ml} / \mathrm{minute}$ ). The FRAX score is calculated for those on prednisone equivalent of $2.5-7.5 \mathrm{mg} / \mathrm{day}$, while those receiving higher doses should increase their major osteoporotic fracture risk by $15 \%$ and by $20 \%$ for the hip. Adapted from Table 1 of the 2017 American College of Rheumatology Guideline for the Prevention and Treatment of Glucocorticoid-Induced Osteoporosis (Buckley et al, Arthritis \& Rheumatology 2017). 
Illustration by Pauline Lu. Abbreviations: GC, glucocorticoids; FRAX, Fracture Risk Assessment Tool (https://www.shef.ac.uk/FRAX/tool.jsp) 